\title{
ARABINOXILANOS Y SU IMPORTANCIA EN LA SALUD
}

\section{TARABINOXYLANS AND THEIR HEALTH EFFECTS}

\section{Ciudad-Mulero, María}

Dpto. Nutrición y Bromatología II. Facultad de Farmacia, Universidad Complutense de Madrid, Madrid, España.mariaciudad@ucm.es

\section{RESUMEN}

Los arabinoxilanos (AX) son los principales polisacáridos no celulósicos en los cereales y forman parte de la fracción soluble de la fibra dietética. Estos compuestos ejercen efectos importantes sobre la salud, siendo el efecto prebiótico uno de los más destacados, el cual, junto con la capacidad antioxidante que han demostrado, convierten a estassustancias en agentes de prevención frente al cáncer de colon.A nivel metabólico los AX actúan como reguladores de la glucemia y del colesterol plasmático, siendo, además, agentes inmunomoduladores. En los últimos años el estudio de los AX ha despertado gran interés puesto que han demostrado tener importantes funciones a nivel fisiológico, funcional y tecnológico.

Palabras clave: arabinoxilanos, arabinoxilooligosacáridos, prebióticos, antioxidante

\begin{abstract}
The arabinoxylans (AX) are the main non cellulosic polysaccharides in cereals being part of the soluble fraction of the dietary fiber. These compounds have important and numerous health benefits. One of the most noteworthy health benefits is their prebiotic effect jointly with their proven antioxidant ability makes these compounds prevention agents of colon cancer diseases. At the metabolic level the AX control glycemic and cholesterol levels being
\end{abstract}


also immunoregulator agents. During the later years the research and interest of the AX has gather considerable attention since they have been related to important functions such as functional, physiological as well as technological.

Keywords: arabinoxylans, arabino-xilo-oligosaccharides, prebiotics, antioxidant

\section{INTRODUCCIÓN}

Los AX son los principales polisacáridos no celulósicos en los cereales (Mendis y Simsek, 2014), están constituidos por una cadena principal lineal de $\beta$-D-(1,4)-xilopiranosasustituida en los grupos hidroxilo de las posiciones 2 y 3 por residuos de tipo L-arabinofuranosil unidos mediante enlaces glucosídicos $\beta(1,4)$.La posición 5 suele estar sustituida por restos de ácido ferúlico (Broekaert et al., 2011; Lafiandra et al., 2014).La hidrólisis enzimática de los AX por las xilanasas y las arabinofuranosidasasproduce arabinoxilooligosacáridos (AXOS) y xilooligosacáridos (XOS) (Adams et al., 2004) que son considerados fibra dietética y que tienen múltiples efectos beneficiosos para la salud (efecto inmunomodulador, hipocolesterolémicos, control de la diabetes tipo 2, mayor absorción de ciertos minerales, efecto prebiótico, etc.) (Mendis y Simsek, 2014).

\section{MATERIAL Y MÉTODOS}

Para la realización del presente trabajo de revisión, se han realizado diferentes búsquedas bibliográficas, en distintas bases de datos científicas como PubMed - NCBI (www. http://www.ncbi.nlm.nih.gov/pubmed), $\quad$ Web $\quad$ OfKnowledge $\quad$ (WOK; https://www.accesowok.fecyt.es), Bucea (www. http://biblioteca.ucm.es) y ScienceDirect (www. sciencedirect.com).

\section{RESULTADOS}

Los AX destacan dentro de la fibra dietética por su efecto funcional, tanto tecnológico como nutricional, proporcionando efectos beneficiosos para la salud de los consumidores.

\section{Efecto prebiótico de los arabinoxilanos}

Para considerarse prebióticos, los AX cumplen con los criterios establecidos por Gibson y Roberfroid (1995). En primer lugar, demuestran su resistencia a la acidez gástrica, a la hidrólisis enzimática y a la absorción gastrointestinal. Van Craeyveld (2009), simuló in vitro

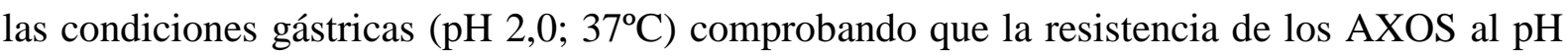
ácido es muy alta. 
En segundo lugar, son fermentables por la microbiota intestinal (Van Craeyveld, 2009). La primera etapa de la degradación de los AX es su hidrólisis por endoxilanasas, donde los fragmentos de AXOS y XOS resultantes son degradados por arabinofuranosidasas y xilosidasas en arabinosa (A) y xilosa (X), a partir de las cuales la microbiota intestinal sintetiza ácidos grasos de cadena corta (AGCC) (Broekaert et al., 2011).

Por último, los AX estimulan selectivamente el crecimiento y/o actividad de las bacterias intestinales beneficiosas para la salud como Lactobacillus cellobiosus, Lactobacillus paracasei y especies de Bifidobacterium, sin estimular el crecimiento de enterococos, Escherichiacoli, Clostridiumdifficiley C. perfringens (Grootaert et al., 2007; Van Craeyveld, 2009; Neyrink et al., 2011).

\section{Efecto sobre el metabolismos lipídico y metabolismo de la glucosa}

Se ha visto que XOS y AXOS son capaces de reducir los niveles plasmáticos de triglicéridos y de colesterol (Saeed et al., 2011; Grooetaert et al., 2007; Neyrink et al., 2011), además, una dieta enriquecida en $\mathrm{AX}$ se relaciona con una menor actividad de la HMG-CoA reductasa hepática (Tong et al., 2014).

En cuanto al control de la glucemia, existen estudios que asocian el consumo de AX con una reducción significativa delos niveles de glucosa postpandrial, de glucemia en ayunas y de la resistencia a la insulina (Lu et al., 2004; Neyrink et al., 2011).

\section{Capacidad antioxidante}

La capacidad antioxidante de los AX está altamente relacionada con el contenido total de fenoles unidos a su estructura. Los compuestos antioxidantes inhiben los radicales libres evitando el estrés oxidativo, la lesión de tejidos y el daño a grandes biomoléculas tales como ADN, lípidos y proteínas (Ayala Soto et al., 2014).

En los alimentos, los AX y los AXOS tienen unidos covalentemente ácido ferúlico, el cual posee propiedades antioxidantes (Niño Medina et al., 2009; Ayala Soto et al., 2014) que han sido confirmadas mediante estudios in vivo realizados con ratas, en las cuales, una vez tratadas con AXOS, se observó una fuerte reducción de la peroxidación lipídica en suero (Broekaert et al., 2011).

\section{Prevención del cáncer de colon}

Los efectos prebióticos de los AX y los AXOS están muy relacionados con su acción protectora frente al cáncer de colon. Se ha visto que reducen la producción bacteriana de $\beta$ glucuronidasa y de amonio en las heces, con lo cual disminuyen los compuestos tóxicos en el colon ejerciendo un papel protector frente a las neoplasias colónicas (Broekaert et al., 2011, Femia et al., 2010; Grootaert et al, 2007). 


\section{Efectos inmunomoduladores}

Existen estudios que indican la acción inmunopotenciadora de los AX, ya que pueden aumentar significativamente la actividad de las células $\mathrm{T}$ y $\mathrm{B}$, mejorando la inmunidad humoral y la inmunidad mediada por células. Los AX también estimulan un aumento en los niveles de IL-2, pudiendo estar relacionado con ciertas propiedades antitumorales (Cao et al., 2011). Además, los AX estimulan la respuesta de anticuerpos, aumentando los niveles de IgG e IgM plasmáticos (Akhtar et al., 2012).

\section{DISCUSIÓN}

Se ha observado que los AX y sus metabolitos ejercen importantes funciones fisiológicas y metabólicas que pueden contribuir a una mejora del estado de salud.

Los AX ejercen efectos protectores frente a enfermedades con elevada prevalencia en las sociedades desarrolladas, por lo tanto constituyen un campo de estudio importante en la actualidad.

\section{CONCLUSIONES}

Los AX son componentes de la fibra que tienen gran importancia en la alimentación ya que reportan numerosos beneficios para la salud.Se ha demostrado que su efecto prebiótico ayuda en la prevención del cáncer de colon. Por otra parte, existen estudios que ponen de manifiesto el importante papel de los AX tanto en el control glucémico, como en el control de la hipercolesterolemia, ya que la ingesta de AX favorece la reducción de los niveles plasmáticos de colesterol y triglicéridos, actuando como factor de prevención de las enfermedades cardiovasculares.También cabe destacar el papel de los AX como moduladores del sistema inmunológico así como la capacidad antioxidante de estos compuestos.

\section{AGRADECIMIENTOS}

Proyecto NºAL 2012-38345 financiado por el Ministerio de Economía y Competitividad.

\section{BIBLIOGRAFÍA}

Adams, EL, Kroon, PA, Williamson, G, Gilbert, HJ, Morris, VJ. 2004. Inactivated enzymes as probes of the structure of arabinoxylans as observed by atomic force microscopy. Carbohydrate Research No 339: 579-590.

Akhtar, M, Tarik, AF, Awais, MM, Iqbal, Z, Muhammad, F, Shahid, M, HiszczynskaSawicka, E. 2012. Studies on wheat bran Arabinoxylan for its immunostimulatory and 
protective effects against avian coccidiosis. Carbohydrate Polymers $\mathrm{N}^{\circ}$ 90: 333-339. doi:10.1016/j.carbpol.2012.05.048.

Ayala-Soto, FE, Serna-Saldívar, SO, García-Lara, S, Pérez-Carrillo, E. 2014. Hydroxycinnamic acids, sugar composition and antioxidant capacityof arabinoxylans extracted from different maize fiber sources. Food Hydrocolloids $\mathrm{N}^{\mathrm{o}}$ 35: 471-475. doi:10.1016/j.foodhyd.2013.07.004

Broekaert, WF, Courtin, CM, Verbeke, K, Van de Wiele, T, Verstraete, W, Delcour, JA. 2011. Prebiotic and Other Health-Related Effects of Cereal-Derived Arabinoxylans, Arabinoxylan-Oligosaccharides, and Xylooligosaccharides. Critical Reviews in Food Science and Nutrition N No 51: 178-194. doi: 10.1080/10408390903044768.

Cao, L, Liu, X, Qian, T, Sun, G, Guo, Y, Chang, F, Zhou, S, Sun, X. 2011. Antitumor and immunomodulatory activity of arabinoxylans: A major constituent of wheat bran. International Journal of Biological Macromolecules $\mathrm{N}^{\circ} \quad 48$ : 160-164. doi:10.1016/j.ijbiomac.2010.10.014.

Femia, AP, Salvadori, M, Broekaert, WF, François, IEJA, Delcour, JA, Courtin, CM, Caderni, G. 2010. Arabinoxylan-oligosaccharides (AXOS) reduce preneoplastic lesions in the colon of rats treated with 1,2-dimethylhydrazine (DMH). Eur. Journal of Nutrition No 49: 127-132. doi: 10.1007/s00394-009-0050-x.

Gibson, GR y Roberfroid, MB. 1995. Dietary modulation of the human colonic microbiota: introducing the concept of prebiotics. Journal of Nutrition N ${ }^{0} 125: 1401-1412$.

Grootaert, C, Delcour, JA, Courtin, CM, Broekaert, WF, Verstraete, W, Van de Wiele, T. 2007. Microbial Metabolism and prebiotic potency of arabinoxylan oligosaccharides in the human intestine. Trends in Food Science \& Technology $\mathrm{N}^{\mathrm{o}}$ 18: 64-71. doi:10.1016/j.tifs.2006.08.004.

Lafiandra, D, Riccardi, G, Shewry, PR. 2014. Improving cereal grain carbohydrates for diet and health. Journal of Cereal Science $N^{\circ}$ 59: 312-326. doi:10.1016/j.jcs.2014.01.001.

Lu, ZX, Walker, KZ, Muir, JG, O’Dea, K. 2004. Arabinoxylan fibre improves metabolic control in people with Type II diabetes. European Journal of Clinical Nutrition $\mathrm{N}^{0} 58$ : 621-628. doi:10.1038/sj.ejcn.1601857.

Mendis, M y Simsek, S. 2014. Arabinoxylans and human health. Food Hydrocolloids $N^{\circ} 42$ : 239-243. doi:10.1016/j.foodhyd.2013.07.022.

Neyrinck, AM, Possemiers, S, Druart, C, Van de Wiele, T, De Backer, F, Cani, PD, Larondelle, Y, Delzenne, NM. 2011. Prebiotic Effects of Wheat Arabinoxylan Related 
to the Increase in Bifidobacteria, Roseburia and Bacteroides/Prevotella in Diet-Induced Obese Mice. PLoS ONE Nº: 1-12. doi: 10.1371/journal.pone.0020944.

Niño-Medina, G, Carvajal-Millán, E, Rascon-Chu, A, Marquez-Escalante, JA, Guerrero, V, Salas-Muñoz, E. 2009. Feruloylated arabinoxylans and arabinoxylans gels: structure, sources and applications. Phytochemistry Review No 9: 111-120. doi: 10.1007/s11101009-9147-3.

Saeed, F, Pasha, I, Anjum, FM, Sultan, MT. 2011. Arabinoxylans and Arabinogalactans: A Comprehensive Treatise. Critical Reviews in Food Science and Nutrition $N^{\circ}$ 51: 467476. doi: 10.1080/10408391003681418.

Tong, LT, Zhong, K, Liu, L, Qiu, J, Guo, L, Zhou, X, Cao, L, Zhou, S. 2014. Effects of dietary wheat bran arabinoxylans on cholesterol metabolism of hypercholesterolemic hamsters. Carbohydrate Polymers No 112: 1-5. doi:10.1016/j.carbpol.2014.05.061.

Van Craeyveld, V. 2009. Tesis Doctoral. Production and functional characterisation of arabinoxylan-oligosaccharides from wheat (Triticum aestivum L.) bran and psyllium (Plantago ovata Forsk) seed husk. Universidad K.U. Leuven, Bélgica. 